\title{
SUFFICIENT CONDITIONS IN THE PROBLEM OF LAGRANGE WITHOUT ASSUMPTIONS OF NORMALCY*
}

\author{
BY \\ MARSTON MORSE
}

Sufficient conditions for an extremal to give a minimum in the ordinary fixed end point problem involve the Jacobi, Weierstrass, and Clebsch conditions. It has been an outstanding problem to establish the corresponding theorem in the problem of Lagrange without assumptions of normalcy or analyticity. Carathéodory [3] reduced the assumptions as to normalcy by introducing the notion of class. More recently Hestenes [4] has employed a similar notion of order of normalcy in dealing with the Jacobi conditions. The paper of Hestenes contains a number of important results independent of the assumption of normalcy.

The present paper establishes sufficient conditions involving the Jacobi, Weierstrass, and Clebsch conditions, employing for the first time, it is believed, no condition of normalcy.

In establishing the desired theorem the writer has come upon a new and powerful method of treating Mayer fields of secondary extremals. This method has also proved the proper tool in attacking other problems not involving a minimum. The fixed end point theorem is treated first and followed by the theorem for the variable end point problem in the modified Bolza [1] form.

The importance of freeing these theorems from the assumptions of normalcy is readily seen upon recalling that the theorems now established lead by simple transformations to corresponding theorems in the Mayer, parametric, and other general forms of the problem, and include earlier theorems of the same general character as special cases.

1. The functional. One is concerned with a set of functions

$$
f(x, y, p), \quad \phi_{\beta}(x, y, p)
$$$$
(\beta=1, \cdots, m)
$$

of the variables

$$
x, \quad(y)=\left(y_{1}, \cdots, y_{n}\right),
$$$$
(p)=\left(p_{1}, \cdots, p_{n}\right)
$$$$
(m<n)
$$

on an open region $R$ of the space of the variables $(x, y, p)$. We suppose the functions (1.1) are of class $C^{3}$ on $R$. Our functional is of the form

\footnotetext{
* Presented to the Society, September 6, 1934; received by the editors July 3, 1934.
} 


$$
J=\int_{a^{1}}^{a^{2}} f\left(x, y, y^{\prime}\right) d x
$$

subject to the conditions

$$
\phi_{\beta}\left(x, y, y^{\prime}\right)=0 \quad(\beta=1, \cdots, m) .
$$

We term an element $\left(x, y, y^{\prime}\right)$ differentially admissible if it satisfies (1.3). An $\operatorname{arc} y_{i}(x)$ is termed differentially admissible if it is of class $D^{1}$ and its elements satisfy (1.3).

We set

$$
F(x, y, p, \lambda)=f+\lambda_{\beta} \phi_{\beta} \quad(\beta=1, \cdots, m) .
$$

By an extremal we mean an arc of class $C^{2}$ together with multipliers $\lambda_{\beta}(x)$ of class $C^{1}$ which satisfy the conditions

$$
\frac{d}{d x} F_{p_{i}}-F_{y_{i}}=0, \quad\left(y^{\prime}\right)=(p) \quad(i=1, \cdots, n),
$$

and the conditions (1.3). We suppose $g$ is such an extremal and is of the form

$$
y_{i}=\bar{y}_{i}(x), \quad \lambda_{\beta}=\bar{\lambda}_{\beta}(x)
$$

for $x$ on an interval

$$
a^{1} \leqq x \leqq a^{2} .
$$

It will frequently be convenient to suppose that $g$ is an inner segment of a slightly longer extremal. By an admissible arc we mean (in $\$ \$ 1,2,3,4)$ a differentially admissible arc which joins the end points of $g$. We shall enumerate the conditions under which $g$ affords a minimum to $J$ relative to neighboring admissible arcs.

It will be convenient to evaluate certain functions along $g$, that is, to set

$$
[x, y, p, \lambda]=\left[x, \bar{y}(x), \bar{y}^{\prime}(x), \bar{\lambda}(x)\right] .
$$

We shall indicate such an evaluation by adding the superscript 0 to the function involved.

We assume that

$$
F_{p_{i} p_{j}}^{0} z_{i} z_{j}>0
$$$$
(i, j=1, \cdots, n)
$$

for each point $x$ on $g$ and set $(z) \neq(0)$ for which

$$
\phi_{\beta p_{i}}^{0} z_{i}=0
$$$$
(\beta=1, \cdots, m) .
$$

We term this condition the Clebsch $S$-condition. 
One sets

$$
E\left[x, y, y^{\prime}, \lambda, Y^{\prime}\right]=F\left(x, y, Y^{\prime}, \lambda\right)-F\left(x, y, y^{\prime}, \lambda\right)-\left(Y_{i}^{\prime}-y_{i}^{\prime}\right) F_{p_{i}}\left(x, y, y^{\prime}, \lambda\right) .
$$

We assume that

$$
E\left(x, y, y^{\prime}, \lambda, Y^{\prime}\right)>0
$$

for each set $\left(x, y, y^{\prime}, \lambda\right)$ in a neighborhood of the sets $\left(x, \bar{y}, \bar{y}^{\prime}, \bar{\lambda}\right)$ on $g$ and for arbitrary sets $\left(Y^{\prime}\right)$, provided merely the sets $\left(x, y, y^{\prime}\right)$ and $\left(x, y, Y^{\prime}\right)$ are differentially admissible and distinct. We term this condition the Weierstrass $S$-condition.

To define the third condition we set

$$
\begin{array}{lrl}
2 \omega\left(\eta, \eta^{\prime}\right) & =F_{y_{i} y_{j} \eta_{i} \eta_{j}}^{0}+2 F_{y_{i} p_{j} \eta_{i} \eta_{j}^{\prime}}^{0}+F_{p_{i} p_{j} \eta_{i}^{\prime} \eta_{j}^{\prime}}^{0} & (i, j=1, \cdots, n), \\
\Phi_{\beta}\left(\eta, \eta^{\prime}\right)=\phi_{\beta p_{i}}^{0} \eta_{i}^{\prime}+\phi_{\beta y_{i}}^{0} \eta_{i} & (\beta=1, \cdots, m) .
\end{array}
$$

The functional

$$
I=\int_{a^{2}}^{a^{2}} 2 \omega\left(\eta, \eta^{\prime}\right) d x
$$

subject to the conditions

$$
\Phi_{\beta}\left(\eta, \eta^{\prime}\right)=0 \quad(\beta=1, \cdots, m),
$$

is termed the second variation. One sets

$$
\Omega\left(\eta, \eta^{\prime}, \mu\right)=\omega+\mu_{\beta} \Phi_{\beta} .
$$

The Euler equations corresponding to the second variation take the form

$$
\frac{d}{d x} \Omega_{\eta_{i^{\prime}}}-\Omega_{\eta_{i}}=0, \quad \Phi_{\beta}=0 .
$$

The corresponding extremals

$$
\eta_{i}=\eta_{i}(x), \quad \mu_{\beta}=\mu_{\beta}(x)
$$

are termed secondary extremals.

It is convenient to set

$$
\Omega_{\eta^{\prime}}=\zeta_{i}, \quad \Phi_{\beta}=0 .
$$

For each value of $x$ the equations (1.8) serve as a transformation from the variables $\left(\eta, \eta^{\prime}, \mu\right)$ to the variables $(\eta, \zeta)$. In particular the secondary extremal (1.7) can be represented in the form

$$
\eta_{i}=\eta_{i}(x), \quad \zeta_{i}=\zeta_{i}(x) .
$$


If the components $\eta_{i}(x)$ of a secondary extremal all vanish for two distinct values of $x$, say $a$ and $b$, but are not all identically null between $a$ and $b$, the values $a$ and $b$ are termed conjugate.

We shall assume that there is no value on the interval $a^{1}<x \leqq a^{2}$ conjugate to $a^{1}$. We term this condition the Jacobi S-condition.

2. Anormal secondary extremals. Secondary extremals for which all the components $\eta_{i}(x)$ are identically null on an interval $(a, b)$ will be termed anormal on $(a, b)$. Other secondary extremals will be termed normal on $(a, b)$. In particular the solution $\eta_{i} \equiv \zeta_{i} \equiv 0$ is anormal.

Let $\alpha$ be a number on the interval $a^{1}<\alpha \leqq a^{2}$. Let $N(\alpha)$ be a set of secondary extremals for which $\eta_{i}\left(a^{1}\right)=0$ and which contains the maximum number of such secondary extremals independent of secondary extremals which are anormal on $\left(a^{1}, \alpha\right)$. All secondary extremals for which $\eta_{i}\left(a^{1}\right)=0$ will be linearly dependent on extremals anormal on $\left(a^{1}, \alpha\right)$ and extremals of $N(\alpha)$.

Let $\pi(\alpha)$ be the number of extremals in $N(\alpha)$. We observe that $\pi(\alpha)$ is monotonically increasing. There will accordingly exist at most a finite set of values of $\alpha$, say $\alpha_{1}, \cdots, \alpha_{r}$, such that

$$
a^{1}<\alpha_{1}<\cdots<\alpha_{r}<a^{2}
$$

at which $\pi(\alpha)$ is discontinuous. The integer $r$ may in particular be null. We set

$$
a^{1}=\alpha_{0}, \quad a^{2}=\alpha_{r+1}
$$

and

$$
N\left(\alpha_{h}\right)=N_{h}, \quad \pi\left(\alpha_{h}\right)=\pi_{h} \quad(h=1, \cdots, r+1) .
$$

There will exist a set $M_{h}$ of $n-\pi_{h}$ secondary extremals which are anormal on $\left(a^{1}, \alpha_{h}\right)$ which with the extremals of $N_{h}$ form a set $A_{h}$ of $n$ independent secondary extremals on which $(\eta)=(0)$ at $a^{1}$. On the $k$ th extremal of the set $A_{h}$ suppose that

$$
\gamma_{i}\left(a^{1}\right)=b_{i k} \quad(k=1, \cdots, n) .
$$

We suppose that the first $n-\pi_{h}$ extremals of the set $A_{h}$ form the set $M_{h}$. Without loss of generality we can also suppose that the columns of the matrix $\left\|b_{i k}\right\|$ have been normed and orthogonalized. We introduce a set $M_{h}^{\prime}$ of $n-\pi_{h}$ secondary extremals of which the $k$ th satisfies the conditions (Hestenes [4], §5)

$$
\eta_{i}\left(a^{1}\right)=b_{i k}, \quad \zeta_{i}\left(a^{1}\right)=0 \quad\left(k=1, \cdots, n-\pi_{h}\right) .
$$

A set of $n$ secondary extremals which are independent and mutually con- 
jugate in the sense of von Escherich is called a conjugate base. The extremals of $N_{h}$ and $M_{h}^{\prime}$ together form a conjugate base $S_{h}$. Let the extremals of $S_{h}$ be represented by the columns of the matrix

$$
\left\|\begin{array}{c}
\eta_{i j}^{h}(x) \\
\zeta_{i j}^{h}(x)
\end{array}\right\| \quad(i, j=1, \cdots, n) .
$$

The determinant

$$
D_{h}(x)=\left|\eta_{i j}^{h}(x)\right|
$$

is called the determinant of the conjugate base. In (2.3) it will be convenient to suppose that the columns which represent extremals of $M_{h}^{\prime}$ come first.

We shall prove the following lemma.

Lemma 2.1. The determinant of the conjugate base $S_{h}$ vanishes at no point on the interval

$$
\alpha_{h-1}<x \leqq \alpha_{h} .
$$

Let

$$
\eta_{i}(x) \equiv c_{j}{ }^{h}{ }_{i j}(x)
$$$$
(i, j=1, \cdots, n)
$$

be an arbitrary linear combination of the columns of the determinant (2.3)'. We suppose that $\eta_{i}(x)$ vanishes at some point $x_{0}$ on the interval (2.4), and shall prove that the constants $c_{i}$ are then all null.

Let $\bar{\zeta}_{i}(x)$ represent the components $\dot{\zeta}_{i}$ of the $k$ th anormal extremal of the set $M_{h}$. One has the integral

$$
\eta_{i} \bar{\zeta}_{i} \equiv \text { const. }
$$

Upon making use of (2.5) and of the fact that the constants (2.2) are normed and orthogonalized we find that (Hestenes [4], \$5),

$$
\eta_{i}\left(a^{1}\right) \bar{\zeta}_{i}\left(a^{1}\right) \equiv c_{k} \equiv \eta_{i}(x) \bar{\zeta}_{i}(x) \text {. }
$$

We infer that $\eta_{i}(x)$ in (2.5) can vanish only if

$$
c_{k}=0 \quad\left(k=1, \cdots, n-\pi_{k}\right) .
$$

The columns involved in (2.5) thus belong at most to extremals of $N_{h}$, and in particular in (2.5), $\eta_{i}\left(a^{1}\right)=0$ for each value of $i$. But $\eta_{i}\left(x_{0}\right)=0$ where $x_{0}$ is on the interval (2.4). We infer that

$$
\eta_{i}(x) \equiv 0 \quad\left(a^{1} \leqq x \leqq x_{0}\right) \quad(i=1, \cdots, n)
$$


since $x_{0}$ is not a conjugate point of $a^{1}$.

We can show that

$$
\eta_{i}(x) \equiv 0 \quad\left(a^{1} \leqq x \leqq \alpha_{h}\right) .
$$

To that end let $\gamma$ represent the secondary extremal obtained by combining the extremals of $N_{h}$ with the same constants as are used in (2.5). If $c$ were the maximum value of $x$ such that $\eta_{i}(x) \equiv 0$ on the interval $\left(a^{1}, c\right)$, and $c<\alpha_{h}$, the function $\pi(\alpha)$ would be discontinuous at $c$. In fact for $\alpha>c$, the set $N(\alpha)$ could be taken as one which included the extremals of $N(c), \gamma$, and possibly other extremals. We infer that (2.7) holds as stated.

But according to the nature of $N_{h}$ the identity (2.7) is valid only if all constants $c_{i}$ in (2.5) are null. The lemma follows directly.

3. Curves which are admissible relative to a conjugate family. Let $K$ be a set of $n$ independent mutually conjugate secondary extremals, and let $L$ denote the set of all extremals linearly dependent on the extremals of $K$. Let the extremals of $L$ be represented by giving their components $\eta_{i}$ and multipliers $\mu_{\beta}$ as follows:

$$
\begin{aligned}
\eta_{i}=c_{j} \eta_{i j}(x) & (i, j=1, \cdots, n), \\
\mu_{\beta}=c_{j} \mu_{\beta j}(x) & (\beta=1, \cdots, m) .
\end{aligned}
$$

We make no assumption concerning the vanishing of the determinant $\left|\eta_{i j}(x)\right|$. By the Hilbert integral belonging to $L$ we mean a line integral in the space of the variables

$$
(x, c)=\left(x, c_{1}, \cdots, c_{n}\right)
$$

of the form

$$
H=\int\left(\Omega-\Omega_{\eta_{i^{\prime}} \eta_{i}^{\prime}}\right) d x+\Omega_{\eta_{i^{\prime}}} d \eta_{i}
$$

in which the variables $\eta_{i}, \mu_{\beta}$ are to be replaced by the respective right members of (3.1) and in which we set

$$
\begin{aligned}
\eta_{i}^{\prime} & =c_{j} \eta_{i j}^{\prime}(x), \\
d \eta_{i} & =\eta_{i j}(x) d c_{j}+c_{i} \eta_{i j}^{\prime}(x) d x .
\end{aligned}
$$

The Hilbert integral will thus take the form

$$
\boldsymbol{H}=\int A(x, c) d x+B_{i}(x, c) d c_{i} .
$$

The variables $(c)$ are arbitrary and $x$ lies on the interval $\left(a^{1}, a^{2}\right)$. That the integral $H$ is independent of the path in the space $(x, c)$ follows in the usual 
way from the fact that the members of the set $K$ are mutually conjugate extremals.

The equations

$$
\eta_{i}=c_{j} \eta_{i j}(x)
$$

define a transformation from the space $(x, c)$ to the space $(x, \eta)$. Let

$$
c_{j}=c_{j}(x)
$$

represent a curve of class $D^{1}$ in the space $(x, c)$. The image under (3.3) of a curve of the form (3.4) will be termed a curve in the space $(x, \eta)$ which is admissible relative to $L$. This curve will be of class $D^{1}$, but not every curve of class $D^{1}$ in the space $(x, \eta)$ will in general be the image of a curve in the space $(x, c)$ of the form (3.4), as examples would show.

We can however prove the following theorem.

THEOREM 3.1. If the Clebsch $S$-condition holds, any segment $\gamma$ of a secondary extremal of a conjugate family $L$ affords a minimum to the second variation relative to curves $\lambda$ which join $\gamma$ 's end points and are admissible relative to $L$.

In the space $(x, c), \gamma$ is represented by a straight line $\gamma_{0}$ on which $x$ alone varies, while $\lambda$ is represented by a curve $\lambda_{0}$ of the form (3.4). The curve $\gamma_{0}$ does not necessarily join the end points of $\lambda_{0}$ in the space $(x, c)$. If in particular the first end points of $\gamma_{0}$ and $\lambda_{0}$ are distinct, these end points can be joined in the space $(x, c)$ by a straight line $p$ on which $x$ is constant. The line $p$ will correspond under (3.3) to the common first end point of $\gamma$ and $\lambda$. Along $p$ the Hilbert integral $H$ will be null. It follows that $H$ has the same value along $\gamma_{0}$ as along $\lambda_{0}$.

Proceeding formally as in the case of ordinary Mayer fields, one sees that

$$
\Delta I=I_{\lambda}-I_{\gamma}=\int_{a^{1}}^{a^{2}} E_{2}\left(x, \eta, \eta^{\prime}, \mu, \bar{\eta}^{\prime}\right) d x
$$

where $E_{2}$ is the Weierstrass $E$-function for the second variation, with

$$
\begin{array}{rlr}
\eta_{i}=c_{j}(x) \eta_{i j}(x), & \eta_{i}^{\prime}=c_{j}(x) \eta_{i j}{ }^{\prime}(x) & (i, j=1, \cdots, n), \\
\mu_{\beta}=c_{j}(x) \mu_{\beta j}(x) & (\beta=1, \cdots, m)
\end{array}
$$

therein, and with $\bar{\eta}_{i}^{\prime}$ taken as the $i$ th slope of the curve $\lambda$ at the point $x$ on $\lambda$. From the fact that the Clebsch $S$-condition holds it follows that $E_{2}$ is never negative for differentially admissible sets $\left(x, \eta, \eta^{\prime}\right)$ and $\left(x, \eta, \bar{\eta}^{\prime}\right)$. We conclude that

$$
\Delta I \geqq 0,
$$


and the theorem is proved.

We add the following lemma.

LEMMA 3.1. There exists a positive constant $\delta$ so small that any segment of a secondary extremal $\gamma$ on which $a \leqq x \leqq a+\delta$, where $a$ is on the interval $\left(a^{1}, a^{2}\right)$, affords a proper minimum to the second variation relative to differentially admissible curves of class $D^{1}$ which join $\gamma^{\prime}$ s end points.

The proof of this lemma is readily given upon setting up a Mayer field of secondary extremals containing $\gamma$. Cf. Morse [7], Lemma 3 and Theorem 4 . Such a Mayer field exists for $x$ on the interval $(a, a+\delta)$ provided $\delta$ is a sufficiently small positive constant.

4. Fixed end points, sufficient conditions. We continue with the following lemma.

LEMMA 4.1. In order that the second variation be non-negative for differentially admissible curves which join the end points of the segment $\left(a^{1}, a^{2}\right)$ of the $x$ axis, it is sufficient that the Clebsch and Jacobi S-conditions hold along $g$.

We return to the notation of $\S 2$, and in particular to the constants $\alpha_{k}$.

(e) If $d$ is a sufficiently small positive constant, any secondary extremal $\gamma$ on which $x$ varies on an interval of the form

$$
a^{1} \leqq x \leqq \alpha_{k}+d \quad(k=0,1, \cdots, r+1)
$$

and on which $(\eta)=(0)$ when $x=a^{1}$, affords a minimum to the second variation relative to differentially admissible curves of class $D^{1}$ which join its end points.

To prove (e) we turn to the conjugate base

$$
S_{h} \quad(h=1, \cdots, r+1)
$$

of $\S 2$, and recall that the determinant $D_{h}(x)$ of this base does not vanish on the interval (2.4). There accordingly exists a positive constant $d_{0}$ independent of $h$ such that $D_{h}(x)$ does not vanish on the interval

$$
\alpha_{h-1}<x \leqq \alpha_{h}+d_{0} \text {. }
$$

We suppose moreover that $d_{0}$ is less than the constant $\delta$ of Lemma 3.1. Statement (e) is valid if we set $d=d_{0}$, as we shall now prove.

Statement (e) is valid if $k=0$ by virtue of Lemma 3.1. Proceeding inductively we shall assume that (e) holds for $d=d_{0}$ and $k=h-1$, and shall prove that (e) holds for $d=d_{0}$ and $k=h$.

Let $\gamma$ be a secondary extremal on which $(\eta)=(0)$ when $x=a^{1}$, and on which $x$ varies on the interval (4.1) for $k=h$. Let $\lambda$ be a differentially admissible curve which joins the end points of $\gamma$. Let $a$ be the segment of $\lambda$ on which 


$$
a^{1} \leqq x \leqq \alpha_{h-1}+d_{0}
$$

and $b$ the remaining segment of $\lambda$, so that we can write

$$
\lambda=a+b .
$$

Now any differentially admissible arc whose end points are not conjugate can be joined by an arc $\eta_{i}(x)$ belonging to a secondary extremal (Hestenes [4], Lemma 7.2). With this understood let $\tau$ be an arc $\eta_{i}(x)$ belonging to a secondary extremal and joining the end points of $a$. We introduce the curve

$$
\mu=\tau+b \text {. }
$$

The curve $\mu$ joins the end points of $\gamma$.

We are assuming that (e) holds for $d=d_{0}$ and $k=h-1$. Hence

$$
I_{a} \geqq I_{\tau} \text {. }
$$

It follows that

$$
I_{a+b} \geqq I_{\tau+b} .
$$

We shall now establish the inequality

$$
I_{\tau+b} \geqq I_{\gamma} .
$$

To that end we represent curves $\eta_{i}(x)$ belonging to secondary extremals dependent on $S_{h}$ in the form

$$
\eta_{i}=c_{i} \eta_{i j}^{h}(x) \text {. }
$$

We regard (4.7) as defining a transformation from the space $(x, c)$ to the space $(x, \eta)$. This transformation is non-singular for $x$ on the interval (4.2). The curve $b$ is accordingly the image in the space $(x, c)$ of a uniquely defined curve

$$
c_{j}=c_{j}(x)
$$

$$
(j=1, \cdots, n)
$$

of class $D^{1}$ on the interval

$$
\alpha_{h-1}+d_{0} \leqq x \leqq \alpha_{h}+d_{0} .
$$

Let $c_{j}{ }^{0}$ be the value of $c_{j}(x)$ when $x=\alpha_{h-1}+d_{0}$. For $x$ on the interval (4.3), $\tau$ will coincide with the curve of the family (4.7) determined by the constants $c_{j}{ }^{0}$. The curve $\tau$ is accordingly the image under the transformation (4.7) of the straight line

$$
c_{j}=c_{j}{ }^{0} \quad\left(a^{1} \leqq x \leqq \alpha_{h-1}+d_{0}\right)
$$

in the space $(x, c)$. The curve $\tau+b$ is thus admissible relative to the conjugate family determined by $S_{h}$. The inequality (4.6) follows from Theorem 3.1. 
Combining (4.5) and (4.6) we find that

$$
I_{a+b} \geqq I_{\gamma} .
$$

Statement (e) is thereby proved.

The lemma is a consequence of statement (e) in the case where $k=r+1$.

It will follow from the next lemma that under the conditions of Lemma 4.1 the second variation is positive definite.

Leмma 4.2. If the Clebsch and Jacobi S-conditions hold along $g$, there exists a conjugate base of secondary extremals whose determinant does not vanish on the interval $a^{1} \leqq x \leqq a^{2}$.

The proof of this lemma is nearly the same as the proof of Theorem 3, Morse [7].

We start with the conjugate base $S_{r+1}$ of $\S 2$. The determinant formed from this base does not vanish at $x=a^{2}$. We can accordingly choose a base

$$
\left\|\begin{array}{l}
\eta_{i j}(x) \\
\zeta_{i j}(x)
\end{array}\right\| \quad(i, j=1, \cdots, n)
$$

for members of the family in which the $j$ th column represents a member of the family such that

$$
\stackrel{2}{\eta_{i j}}=\delta_{i}^{i} .
$$

The superscripts 1 and 2 are used to indicate evaluation for $x=a^{1}$ and $x=a^{2}$ respectively. We introduce a second conjugate family $H$ with base $B$ of the form

$$
\left\|\begin{array}{l}
\bar{\eta}_{i j}(x) \\
\bar{\zeta}_{i j}(x)
\end{array}\right\|
$$

and such that

$$
\dot{\bar{\eta}}_{i j}^{2}=\delta_{i}^{i}, \quad \bar{\zeta}_{i j}^{2}=\zeta_{i j}^{2}-\delta_{i}^{j} .
$$

We represent the family $H$ in the form

$$
\begin{aligned}
& \eta_{i}=u_{j} \bar{\eta}_{i j}(x), \\
& \zeta_{i}=u_{j} \bar{\zeta}_{i j}(x),
\end{aligned}
$$

where the symbols $u_{j}$ represent constants.

The conjugate base $B$ will serve as the conjugate base whose existence is affirmed in the lemma. 
To establish the truth of this statement we assume that it is false, and hence that the determinant $\left|\bar{\eta}_{i j}\right|$ vanishes at some point $x=c$ on the interval

$$
a^{1} \leqq x<a^{2} .
$$

There will then exist an extremal $\gamma$ of $H$ determined by a set of constants $(u) \neq(0)$ such that $\eta_{i}(c)=0$ on $\gamma$ for each $i$. Let $\lambda$ be a curve which consists of the $x$ axis from $x=a^{1}$ to $x=c$ and of the curve $\eta_{i}=\eta_{i}(x)$ belonging to $\gamma$ from $x=c$ to $x=a^{2}$. Upon evaluating the second variation $I$ along $\lambda$ from $x=a^{1}$ to $x=a^{2}$ we find that

$$
I_{\lambda}=\zeta_{i j}^{2} u_{i} u_{j}-u_{i} u_{i} .
$$

On the other hand there will be a secondary extremal $\mu$ dependent on the base $S_{r+1}$ for which the curve $\eta_{i}(x)$ joins the end points of $\lambda$. This extremal will have the form

$$
\eta_{i}=\eta_{i j}(x) u_{j}, \quad \zeta_{i}=\zeta_{i j}(x) u_{j}
$$

and we see that

$$
I_{\mu}=\zeta_{i j}^{2} u_{i} u_{j} .
$$

It follows from statement (e) of the proof of the preceding lemma that $I_{\lambda} \geqq I_{\mu}$. But this is impossible since $(u) \neq(0)$.

We infer that the determinant $\left|\bar{\eta}_{i j}(x)\right|$ does not vanish on the interval (4.10). The base $B$ will thus serve as the base of the lemma, and the proof is complete.

We come to a basic theorem.

THEOREM 4.1. In order that the extremal $g$ afford a proper minimum to $J$ relative to neighboring admissible curves, it is sufficient that the Clebsch, Jacobi, and Weierstrass $S$-conditions hold along $g$.

The conjugate family of secondary extremals whose existence is affirmed in the last lemma forms a Mayer field of secondary extremals covering a neighborhood of the segment $\left(a^{1}, a^{2}\right)$ of the $x$ axis. This family can be used as in Morse [7], Theorem 4, to establish the existence of a Mayer field of primary extremals including $g$ and covering a neighborhood of $g$.

The theorem follows in the usual manner.

5. General end conditions. We turn to the problem under general end conditions. The preceding results lead to a set of sufficient conditions involving the Jacobi condition which make no assumption concerning normalcy. 
The form of the problem is a modification of the Bolza problem introduced by the author (Morse [8]). We suppose that we have an extremal $g$ as before. Points near the first and last end points of $g$ will be denoted by $\left(x^{1}, y^{1}\right)$ and $\left(x^{2}, y^{2}\right)$ respectively. The end conditions have the form

$$
x^{s}=x^{s}(\alpha), \quad y_{i}^{s}=y_{i}{ }^{s}(\alpha)
$$$$
(s=1,2 ; i=1, \cdots, n)
$$

where $(\alpha)$ represents a set of $r$ variables $\alpha_{h}$. The functions $x^{*}(\alpha)$ and $y_{i}{ }^{\circ}(\alpha)$ are assumed to be of class $C^{2}$ for $(\alpha)$ near (0) and to yield the end points of $g$ when $(\alpha)=(0)$. No assumption is made concerning the rank of the matrix of the functions on the right of (5.1). The differential conditions are as before, but the functional $J$ is replaced by the more general functional

$$
J=\theta(\alpha)+\int_{x^{1}(\alpha)}^{x^{2}(\alpha)} f\left(x, y, y^{\prime}\right) d x
$$

in which $\theta(\alpha)$ is a function of $(\alpha)$ of class $C^{2}$ for $(\alpha)$ near (0).

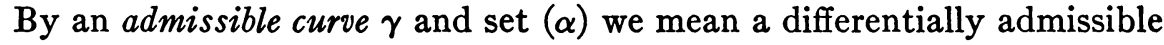
curve $\gamma$ and set $(\alpha)$ such that $\gamma$ satisfies the end conditions with the set $(\alpha)$. The problem is one of determining conditions under which $g$ and the set $(\alpha)=(0)$ afford a minimum to $J$ relative to $J$ 's value for admissible curves $\gamma$ and sets $(\alpha)$ for which $\gamma$ neighbors $g$ and $(\alpha)$ neighbors (0).

We assume that $g$ and the set $(\alpha)=(0)$ satisfy the transversality condition (Morse and Myers [6])

$$
\left[\left(F^{0}-F_{p_{i}}^{0} \bar{y}_{i}^{\prime}\right) d x^{0}+F_{p_{i}}^{0} d y_{i}^{0}\right]_{1}^{2}+d \theta \equiv 0,
$$

as an identity in the differentials $d \alpha_{h}$ in terms of which $d x^{*}, d y_{i}{ }^{\circ}$, and $d \theta$ are to be expressed.

The second variation is the functional (Morse [8])

$$
I=b_{h k} u_{h} u_{k}+\int_{a 1}^{a 2} 2 \omega\left(\eta, \eta^{\prime}\right) d x \quad(h, k=1, \cdots, r),
$$

subject to secondary end conditions of the form

$$
\dot{\eta}_{i}^{\dot{*}}=c_{i h}^{\dot{*}} u_{h} \quad(s=1,2 ; h=1, \cdots, r),
$$

where $b_{h k}$ and $c_{i h}^{s}$ represent constants of which $b_{h k}=b_{k h}$. It is here understood that $x^{1}=a^{1}$ and $x^{2}=a^{2}$. The differential conditions are as before. A curve $\gamma$ and set $(u)$, such that $\gamma$ is differentially admissible and satisfies (5.4) with the set $(u)$, is termed admissible.

For a problem under general end conditions Mayer has stated a sufficiency condition in terms of a quadratic form. Bliss [2] and Hestenes [4] 
have modified this condition. We introduce a further modification of this condition which simplifies its use.

To that end let

$$
\eta_{i p}(x), \quad \zeta_{i p}(x), \quad u_{h p} \quad(i=1, \cdots, n ; h=1, \cdots, r ; p=1, \cdots, q)
$$

be a set of $q$ secondary extremals and constants (u) which satisfy (5.4). Suppose moreover that this set contains the maximum number of admissible secondary extremals and constants $(u)$ which are independent of sets $\eta_{i}(x) \equiv 0, \zeta_{i}(x),(u)=(0)$ belonging to anormal secondary extremals. Each admissible secondary extremal and set $(u)$ is linearly dependent upon the members of the set (5.5) together with an anormal secondary extremal and set $(u)=(0)$. We consider the family

$$
\eta_{i}=v_{p} \eta_{i p}(x), \quad \zeta_{i}=v_{p} \zeta_{i p}(x), \quad u_{h}=v_{p} u_{h p},
$$

of admissible secondary extremals and corresponding sets $(u)$. Upon evaluating $I$ along the member of this family determined by $(v)$ one obtains a quadratic form $H(v)$. By the Mayer $S$-condition we mean the condition that $H(v)$ be positive definite.

The theorem here is as follows.

THEOREM 5.1. In order that the extremal $g$ and set $(\alpha)=(0)$ afford a minimum to $J$ relative to neighboring admissible curves and sets $(\alpha)$ it is sufficient that $g$ and the set $(\alpha)=(0)$ satisfy the transversality condition, that there be no conjugate point of $x=a^{1}$ on the interval $a^{1}<x<a^{2}$, and that the Clebsch, Weierstrass, and Mayer S-conditions hold.

Hestenes [4] has shown that $g$ and the set $(\alpha)=(0)$ afford the desired minimum provided the second variation is positive definite for admissible sets $(\eta)$ and $(u)$. Cf. Currier [5]. The problem here is accordingly to show that the second variation is positive for non-null admissible sets $(\eta)$ and $(u)$.

We observe that $x=a^{2}$ is not conjugate to $x=a^{1}$. For otherwise there would be a secondary extremal for which $\eta_{i} \neq 0$, which would satisfy the end conditions with the the set $(u)=(0)$, and would appear with this $\eta_{i}(x)$ and set $(u)$ as a member of the family (5.6) for which $(v) \neq(0)$. We would then have $H(v)=0$, contrary to hypothesis.

It follows from the preceding sections that each secondary extremal for which $a^{1} \leqq x \leqq a^{2}$ gives a proper minimum to $I$ relative to admissible curves $\eta_{i}(x)$ which join its end points. That $I>0$ for admissible secondary extremals and sets $(u)$ for which $(\eta)$ and $(u)$ are not both null follows from the positive definiteness of $H(v)$. Hence $I$ is positive definite as stated.

The proof of the theorem is complete. 


\section{BIBLIOGRAPHY}

1. Bolza, Über den "anormalen Fall" beim Lagrangeschen und Mayerschen Problem mit gemischten Bedingungen und variablen Endpunkten, Mathematische Annalen, vol. 74 (1913), pp. 430-446.

2. Bliss, The problem of Bolza in the calculus of variations, Annals of Mathematics, vol. 33 (1932), pp. 261-274.

3. Carathéodory, Üeber die Einteilung der Variationsprobleme von Lagrange nach Klassen, Commentarii Mathematici Helvetici, vol. 5 (1933), pp. 1-19.

4. Hestenes, Sufficient conditions for the problem of Bolza in the calculus of variations, these Transactions, vol. 35 (1934).

5. Currier, The variable end point problem of the calculus of variations including a generalization of the classical Jacobi conditions, these Transactions, vol. 34 (1932), pp. 689-704.

6. Morse and Myers, The problems of Lagrange and Mayer with variable end points, Proceedings of the American Academy of Arts and Sciences, vol. 66 (1931), pp. 235-253.

7. Morse, Sufficient conditions in the problem of Lagrange with fixed end points, Annals of Mathematics, vol. 32 (1931), pp. 567-577.

8. Morse, Sufficient conditions in the problem of Lagrange with variable end points, American Journal of Mathematics, vol. 53 (1931), pp. 517-546.

9. Note added in proof. At the September meeting of the Society at Williamstown, Dr. Reid, unaware of the existence of the present paper, reported on a proof of theorems similar to the theorems contained herein. In the final theorems he assumed normalcy on the interval $\left(a^{1}, a^{2}\right)$ as against the author's weaker assumption that a $\lambda_{0}$ exists which is not zero. More recently Dr. Hestenes has announced proofs of the theorems concerned.

HARVARD UNIVERSITY,

Cambridge, Mass. 